\title{
A Bayesian Scoring Technique for Mining Predictive and Non-Spurious Rules
}

\author{
Iyad Batal ${ }^{1}$, Gregory Cooper ${ }^{2}$, and Milos Hauskrecht ${ }^{1}$ \\ 1 Department of Computer Science, University of Pittsburgh \\ 2 Department of Biomedical Informatics, University of Pittsburgh
}

\begin{abstract}
Rule mining is an important class of data mining methods for discovering interesting patterns in data. The success of a rule mining method heavily depends on the evaluation function that is used to assess the quality of the rules. In this work, we propose a new rule evaluation score - the Predictive and Non-Spurious Rules (PNSR) score. This score relies on Bayesian inference to evaluate the quality of the rules and considers the structure of the rules to filter out spurious rules. We present an efficient algorithm for finding rules with high PNSR scores. The experiments demonstrate that our method is able to cover and explain the data with a much smaller rule set than existing methods.
\end{abstract}

\section{Introduction}

The large amounts of data collected today provide us with an opportunity to better understand the behavior and structure of many natural and man-made systems. Rule mining is an important direction of machine learning and data mining research, which aims to elicit knowledge from data in terms of if-then rules that are intuitive and easy to understand by humans.

In this work, we study and apply rule mining to discover patterns in supervised learning tasks, where we have a specific target variable (outcome) and we want to find patterns (subpopulations of data instances) where the distribution of the target variable is statistically "most interesting". Examples of such patterns are: "subpopulation of patients who smoke and have a positive family history are at a significantly higher risk for lung cancer than the rest of the patients". This task has a high practical relevance in many domains of science or business. For example, finding a pattern that clearly and concisely defines a subpopulation of patients that respond better (or worse) to a certain treatment than the rest of the patients can speed up the validation process of this finding and its future utilization in patient-management.

In order to perform supervised rule discovery, we need to define a search algorithm to explore the space of potential rules and a scoring function to assess the interestingness of the rules. In this work, we use Frequent Pattern Mining (FPM) [1] to search for rules. The advantage of FPM is that it performs a more systematic search than heuristic rule induction approaches, such as greedy sequential covering [7, 9]. However, its main disadvantage is that it often produces

P. Flach et al. (Eds.): ECML PKDD 2012, Part II, LNCS 7524, pp. 260-276, 2012.

(C) Springer-Verlag Berlin Heidelberg 2012 
a large number of rules. Moreover, many of these rules are spurious because they can be naturally explained by other simpler (more general) rules. Therefore, it is crucial to devise an effective scoring function that allows us to select important and non-redundant rules from a large pool of frequent patterns.

To achieve this goal, we introduce the Predictive and Non-Spurious Rules (PNSR) score. This score applies Bayesian inference to evaluate the quality of individual rules. In addition, it considers the structure of patterns to assure that every rule is not only predictive with respect to the general population, but also with respect to all of its simplifications (generalizations). We show that using our score to mine the top rules, we are able to cover and explain the data with much fewer rules compared with classical supervised rule discovery methods. Finally, we present an efficient algorithm that integrates rule evaluation with frequent pattern mining and applies pruning strategies to speed up the mining.

\section{Supervised Descriptive Rule Discovery}

In this work, we are interested in applying rule mining in the supervised setting, where we have a special variable of interest $Y$ (the target variable) and we want to mine rules that can help us to uncover "interesting" dependencies between $Y$ and the input variables (attributes).

The dominant paradigm for supervised rule induction is to apply a sequential covering method [7 9], which learns a set of rules by first learning a single rule, removing the positive instances it covers and then repeating the process over the remaining instances. However, this approach is not appropriate for knowledge discovery because the rules are induced from biased data (including only positive instances not covered by previous rules). Therefore, the rules are difficult to interpret and understand by the user.

In contrast to the sequential covering approach, our task is to find a set of comprehensible rules/patterns that are statistically interesting with respect to the entire data, e.g., the rules should have wide coverage and unusual distributional characteristics with respect to the target variable [18]. This task appeared in the literature under a variety of different names, such as contrast set mining [2], emerging pattern mining [11] and subgroup discovery [17, 18]. Later on, 23] provided a unifying framework of this work which is named Supervised Descriptive Rule Discovery (SDRD).

To apply $S D R D$, we need to define a search algorithm to explore the space of potential rules and a scoring function $S$ (quality measure) to assess the interestingness of each rule ( $S$ maps each rule $R_{i}$ to a real number $S\left(R_{i}\right) \in \mathbb{R}$ that reflects its importance). Our objective in this work is to design a function $S$ such that the top rules do not only predict well the target class variable compared to the entire population, but are also non-spurious in that their prediction is better than all of their generalizations (simplifications).

\subsection{Definitions}

Let $D=\left\{x_{i}, y_{i}\right\}_{i=1}^{n}$ be our data, where each instance $x_{i}$ is described by a fixed number of attributes and is associated with a class label $y_{i} \in \operatorname{dom}(Y)$. We assume 
that all attributes have discrete values (numeric attributes must be discretized [13, 28]).

We call every attribute-value pair an item and a conjunction of items a pattern. A pattern that contains $k$ items is called a $k$-pattern. For example, Education $=$ $P h D \wedge$ Marital-status $=$ Single is a 2-pattern .

Pattern $P$ is a subpattern of pattern $P^{\prime}$, denoted as $P \subset P^{\prime}$, if every item in $P$ is contained in $P^{\prime}$ and $P \neq P^{\prime}$. In this case, $P^{\prime}$ is a superpattern of $P$. For example, $P_{1}:$ Education $=P h D$ is a subpattern of $P_{2}:$ Education $=P h D \wedge$ Marital-status $=$ Single. This subpattern (more-general-than) relation defines a partial ordering of patterns, i.e. a lattice structure, as shown in Figure 1.

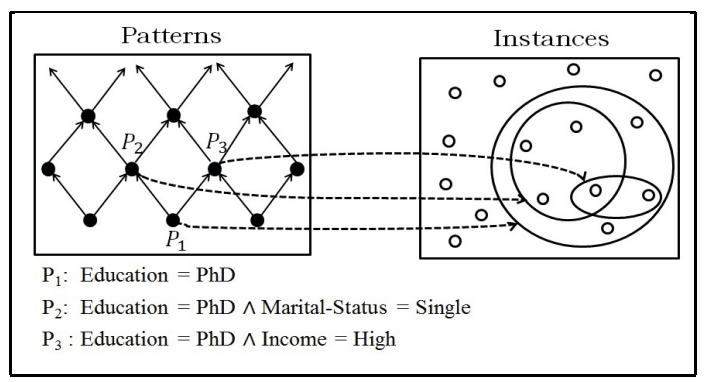

Fig. 1. The box on the left shows the set of all patterns and the box on the right shows the set of all instances. Each pattern is associated with a group of instances that satisfy the pattern. The patterns are organized in a lattice structure according to the subpattern-superpattern relation.

Instance $x_{i}$ satisfies pattern $P$, denoted as $P \in x_{i}$, if every item in $P$ is present in $x_{i}$. Every pattern $P$ defines a group (subpopulation) of the instances that satisfy $P: G_{P}=\left\{\left(x_{i}, y_{i}\right): x_{i} \in D \wedge P \in x_{i}\right\}$. If we denote the empty pattern by $\phi, G_{\phi}$ represents the entire data $D$. Note that $P \subset P^{\prime}\left(P\right.$ is a subpattern of $\left.P^{\prime}\right)$ implies that $G_{P} \supseteq G_{P^{\prime}}$ (see Figure 1).

The support of pattern $P$ in dataset $D$, denoted as $\sup (P, D)$, is the number of instances in $D$ that satisfy $P$ (the size of $G_{P}$ ). Given a user defined minimum support threshold $\sigma, P$ is called a frequent pattern if $\sup (P, D) \geq \sigma$.

A rule is defined as $P \Rightarrow y$, where $P$ (the condition) is a pattern and $y \in$ $\operatorname{dom}(Y)$ (the consequent) is a class label. We say that $P \Rightarrow y$ is a subrule of $P^{\prime} \Rightarrow y^{\prime}$ if $P \subset P^{\prime}$ and $y=y^{\prime}$. The coverage of rule $P \Rightarrow y$ is the proportion of instances in the data that satisfy $P$. The confidence of rule $P \Rightarrow y$, denoted as $\operatorname{conf}(P \Rightarrow y)$, is the proportion of instances from class $y$ among all the instances that satisfy $P$, i.e., it is the maximum likelihood estimation of $\operatorname{Pr}(Y=y \mid P)$.

\subsection{Rule Evaluation}

A straightforward approach to $S D R D$ is to use a rule quality measure (cf [14]) to score each rule by contrasting it to the general population (the entire data) and 
report the top rules to the user. We will argue that this approach is ineffective and can lead to many spurious (redundant) rules. We start by illustrating the spurious rules problem using an example and then describe it more formally in Section 2.3 .

Example 1. Assume our objective is to identify populations of patients who are at high risk of developing coronary heart disease (CHD). Assume our dataset contains 150 instances, 50 of them are CHD cases and the others are controls. That is, the CHD prior, i.e. $\operatorname{conf}(\Phi \Rightarrow C H D)$, is $50 / 150 \approx 33.3 \%$.

Now, our task is to evaluate the following 3 rules:

$-R_{1}$ :Race $=$ White $\Rightarrow$ CHD

[\# cases $=29, \#$ controls $=61$, conf $=32.2 \%$ ]

$-R_{2}$ :Family history $=$ Yes $\Rightarrow \mathrm{CHD}$ [\#cases $=30, \#$ controls $=20$, conf $=60 \%$ ]

$-R_{3}$ :Family history $=$ Yes $\wedge$ Race $=$ White $\Rightarrow \mathrm{CHD}$ [\#cases $=21, \#$ controls $=11$, conf $=65.6 \%]$

For each rule, we show the number of CHD cases and the number of controls that the rule covers. We also show the confidence of the rule.

One of the commonly used approaches to filter out uninteresting rules is to apply the $\chi^{2}$ test to assure that there is a significant positive correlation between the condition and the consequent of each rule [2, 5, 20, 22]. If we apply the $\chi^{2}$ test on our three rules, the p-values we get for $R_{1}, R_{2}$, and $R_{3}$ are $0.724,9.6 \times 10^{-7}$, and $1.2 \times 10^{-5}$, respectively. That is, both $R_{2}$ and $R_{3}$ are statistically (very) significant with respect to a significance level $\alpha=0.05$. Moreover, these two rules will be considered interesting using most rule quality measures [14].

[3] proposed the confidence improvement constraint, which says that each rule in the result should have a higher confidence than all of its subrules:

$$
\operatorname{conf}(P \Rightarrow y)-\max _{S \subset P}\{\operatorname{conf}(S \Rightarrow y)\}>0
$$

This filter have been used quite a lot in the rule mining literature [15, 19, 20, 26]. If we applied the confidence improvement constraint to our working example, both $R_{2}$ and $R_{3}$ will be retained.

As we can see, both $\chi^{2}$ test and confidence improvement agree that $R_{3}$ is an interesting rule. However, this rule may seem predictive only because it contains a simpler predictive rule $\left(R_{2}\right)$. So should we consider $R_{3}$ to be interesting (show it to the user) or spurious? We will revisit this question after introducing the PNSR score.

\subsection{Spurious Rules}

Spurious rules are formed by adding irrelevant items to the antecedent of a simpler predictive rule. Let us illustrate this using the simple Bayesian belief network in Figure 2. In this network, the value of the class variable $Y$ only depends on the value of feature $F_{1}$ and is independent of the values of the other 


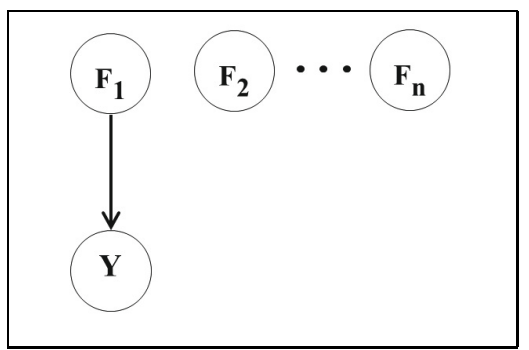

Fig. 2. Illustrating the problem of spurious rules

features: $Y \Perp F_{i}: i \in\{2, \ldots, n\}$. Assume that pattern $P: F_{1}=1$ is predictive of class $Y=y_{1}$, so that $\operatorname{Pr}\left(y_{1} \mid P\right)>\operatorname{Pr}\left(y_{1}\right)$. Clearly, $P$ is the only important pattern for predicting $y_{1}$.

Now consider pattern $P^{\prime}$ that is a superpattern of $P, P^{\prime}: F_{1}=1 \wedge F_{q_{1}}=$ $v_{q_{1}} \wedge \ldots \wedge F_{q_{k}}=v_{q_{k}}$, where $F_{q_{i}} \in\left\{F_{2}, \ldots, F_{n}\right\}$ and $v_{q_{i}}$ is any possible value of variable $F_{q_{i}}$. The network structure implies that $\operatorname{Pr}\left(y_{1} \mid P^{\prime}\right)=\operatorname{Pr}\left(y_{1} \mid P\right)$, hence $\operatorname{Pr}\left(y_{1} \mid P^{\prime}\right)$ is also larger than the prior $\operatorname{Pr}\left(y_{1}\right)$.

The problem is that if we evaluate the rules individually (without considering the nested structure of the patterns), we may falsely think that $P^{\prime} \Rightarrow y_{1}$ is an important rule. However, this rule is totally redundant given its subrule $P \Rightarrow y_{1}$. Even by requiring complex rules to have a higher confidence than their simplifications (the confidence improvement) [3, 15, 19, 20, 26], the problem still exists and many spurious rules can easily satisfy this constraint due to noise in sampling. Clearly, having spurious rules in the results is undesirable because they overwhelm the user and prevent him/her from understanding the real causalities in the data.

\section{Mining Predictive and Non-Spurious Rules}

In this section, we present our approach for scoring/ranking rules. We start by defining a Bayesian score to evaluate the predictiveness of a rule with respect to a more general population. After that, we introduce the PNSR-score to address the problem of spurious rules. Lastly, we present an efficient mining algorithm that integrates rule evaluation with frequent pattern mining.

\subsection{Classical Rule Quality Measures}

A large number of rule quality measures have been proposed in the literature to evaluate the interestingness of individual rules. Examples of such measures include confidence, lift, weighted relative accuracy, J-measure, and others (cf [14]). Most of these measures trade-off two factors: 1) the distributional unusualness of the class variable in the rule compared to the general population and 2) the coverage of the rule, which reflects its generality [18, 23]. This trade-off is often achieved in an ad-hoc way, for instance by simply multiplying these two factors 
as in the weighted relative accuracy score [17] or in the J-measure [25]. Furthermore, most interestingness measures rely on point estimates of these quantities, often using the maximum likelihood estimation, and they do not capture the uncertainty of the estimation. In the following, we present a novel Bayesian score to evaluate the quality of a rule.

\subsection{The Bayesian Score}

Suppose we want to evaluate rule $P \Rightarrow y$ with respect to a group of instances $G$ where $G_{P} \subseteq G$. Intuitively, we would like the rule to get a high score when there is a strong evidence in the data to support the hypothesis that $\operatorname{Pr}\left(Y=y \mid G_{P}\right)>$ $\operatorname{Pr}(Y=y \mid G)$. Our Bayesian score treats these probabilities as random variables as opposed to using their point estimation as in the classical measures [14].

Let us begin by defining $M_{e}$ to be the model that conjectures that all instances in group $G$ have the same probability for having class $Y=y$, even though we are uncertain what that probability is. Let us denote $\operatorname{Pr}(Y=y \mid G)$ by $\theta$. To represent our uncertainty about $\theta$, we use a beta distribution with parameters $\alpha$ and $\beta$. Let $N_{* 1}$ be the number of instances in $G$ with class $Y=y$ and let $N_{* 2}$ be the number of instances in $G$ with class $Y \neq y$. The marginal likelihood for model $M_{e}$ is as follows:

$$
\operatorname{Pr}\left(G \mid M_{e}\right)=\int_{\theta=0}^{1} \theta^{N_{* 1}} \cdot(1-\theta)^{N_{* 2}} \cdot \operatorname{beta}(\theta ; \alpha, \beta) d \theta
$$

The above integral yields the following well known closed-form solution [16] :

$$
\operatorname{Pr}\left(G \mid M_{e}\right)=\frac{\Gamma(\alpha+\beta)}{\Gamma\left(\alpha+N_{* 1}+\beta+N_{* 2}\right)} \cdot \frac{\Gamma\left(\alpha+N_{* 1}\right)}{\Gamma(\alpha)} \cdot \frac{\Gamma\left(\beta+N_{* 2}\right)}{\Gamma(\beta)}
$$

where $\Gamma$ is the gamma function.

Let us define $M_{h}$ to be the model that conjectures that the probability of $Y=y$ in $G_{P}$, denoted by $\theta_{1}$, is different from the probability of $Y=y$ in the instances of $G$ not covered by $P\left(G \backslash G_{P}\right)$, denoted by $\theta_{2}$. Furthermore, $M_{h}$ believes that $\theta_{1}$ is higher than $\theta_{2}$. To represent our uncertainty about $\theta_{1}$, we use a beta distribution with parameters $\alpha_{1}$ and $\beta_{1}$, and to represent our uncertainty about $\theta_{2}$, we use a beta distribution with parameters $\alpha_{2}$ and $\beta_{2}$. Let $N_{11}$ and $N_{12}$ be the number of instances in $G_{P}$ with $Y=y$ and with $Y \neq y$, respectively. Let $N_{21}$ and $N_{22}$ be the number of instances outside $G_{P}$ with $Y=y$ and with $Y \neq y$, respectively (see Figure 3). Note that $N_{* 1}=N_{11}+N_{21}$ and $N_{* 2}=N_{12}+N_{22}$.

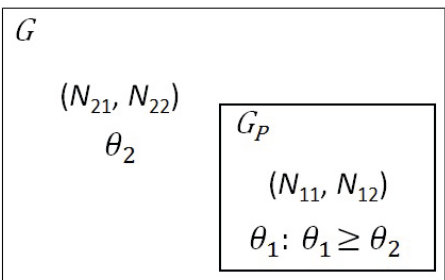

Fig. 3. A diagram illustrating model $M_{h}$ 
The marginal likelihood for model $M_{h}$ is defined as follows:

$$
\begin{gathered}
\operatorname{Pr}\left(G \mid M_{h}\right)=\int_{\theta_{1}=0}^{1} \int_{\theta_{2}=0}^{\theta_{1}} \theta_{1}^{N_{11}} \cdot\left(1-\theta_{1}\right)^{N_{12}} \cdot \theta_{2}^{N_{21}} \cdot\left(1-\theta_{2}\right)^{N_{22}} \\
\cdot \frac{\operatorname{beta}\left(\theta_{1} ; \alpha_{1}, \beta_{1}\right) \cdot \operatorname{beta}\left(\theta_{2} ; \alpha_{2}, \beta_{2}\right)}{k} d \theta_{2} d \theta_{1}
\end{gathered}
$$

where $k$ is a normalization constant for the parameter prior 1 . Note that this formula does not assume that the parameters are independent, but rather constrains $\theta_{1}$ to be higher than $\theta_{2}$.

Below we show the closed form solution we obtained by solving Equation 2. The derivation of the solution is omitted in this manuscript due to space limitation 2 .

$$
\begin{aligned}
\operatorname{Pr}\left(G \mid M_{h}\right)= & \frac{1}{k} \cdot \frac{\Gamma\left(\alpha_{1}+\beta_{1}\right)}{\Gamma\left(\alpha_{1}\right) \Gamma\left(\beta_{1}\right)} \cdot \frac{\Gamma\left(\alpha_{2}+\beta_{2}\right)}{\Gamma\left(\alpha_{2}\right) \Gamma\left(\beta_{2}\right)} . \\
& \sum_{j=a}^{a+b-1}\left(\frac{\Gamma(a) \Gamma(b)}{\Gamma(j+1) \Gamma(a+b-j)} \cdot \frac{\Gamma(c+j) \Gamma(a+b+d-j-1)}{\Gamma(a+b+c+d-1)}\right)
\end{aligned}
$$

where $a=N_{21}+\alpha_{2}, b=N_{22}+\beta_{2}, c=N_{11}+\alpha_{1}, d=N_{12}+\beta_{1}$. We solve for $k$ by applying Equation 3 (without the $k$ term) with $a=\alpha_{2}, b=\beta_{2}, c=\alpha_{1}$ and $d=\beta_{1}$.

Equation 3 can be expressed in logarithmic form (to avoid computing very large numbers). Its computational complexity is $O(b)=O\left(N_{22}+\beta_{2}\right)$ (the number of terms in the summation). It turns out that we can redefine the solution of Equation 2 so that its computational complexity is $O\left(\min \left(N_{11}+\alpha_{1}, N_{12}+\beta_{1}, N_{21}+\right.\right.$ $\left.\alpha_{2}, N_{22}+\beta_{2}\right)$ ). The modifications that achieve this complexity result are omitted due to space limitation 3 .

Lastly, let $M_{l}$ be the model that conjectures that $\theta_{1}$ is lower than $\theta_{2}$. The marginal likelihood for $M_{l}$ is similar to Equation 2, but integrates $\theta_{2}$ from 0 to 1 and constrains $\theta_{1}$ to be integrated from 0 to $\theta_{2}$ (forcing $\theta_{1}$ to be smaller than $\left.\theta_{2}\right)$. The solution for $P\left(G \mid M_{l}\right)$ can reuse the terms computed in Equation 3 and can be computed with complexity $\mathrm{O}(1)$.

Now that we computed the marginal likelihood for models $M_{e}, M_{h}$ and $M_{l}$, we compute the posterior probability of $M_{h}$ (the model of interest) using Bayes theorem:

$$
\operatorname{Pr}\left(M_{h} \mid G\right)=\frac{\operatorname{Pr}\left(G \mid M_{h}\right) \operatorname{Pr}\left(M_{h}\right)}{\operatorname{Pr}\left(G \mid M_{e}\right) \operatorname{Pr}\left(M_{e}\right)+\operatorname{Pr}\left(G \mid M_{h}\right) \operatorname{Pr}\left(M_{h}\right)+\operatorname{Pr}\left(G \mid M_{l}\right) \operatorname{Pr}\left(M_{l}\right)}
$$

To be "non-informative", we might simply assume that all three models are equally likely a-priori: $\operatorname{Pr}\left(M_{e}\right)=\operatorname{Pr}\left(M_{h}\right)=\operatorname{Pr}\left(M_{l}\right)=\frac{1}{3}$.

Equation 4 quantifies in a Bayesian way how likely (a posteriori) is the model which presumes $\operatorname{Pr}\left(Y=y \mid G_{P}\right)$ is higher than $\operatorname{Pr}(Y=y \mid G)$. Since this is the

\footnotetext{
${ }^{1} k=\frac{1}{2}$ if we use uniform priors on both parameters by setting $\alpha_{1}=\beta_{1}=\alpha_{2}=\beta_{2}=1$.

2 The derivation can be found on the author's website: www.cs.pitt.edu/ iyad.

3 These modifications can found on the author's website: www.cs.pitt.edu/ iyad.
} 
quantity we are interested in, we use $\operatorname{Pr}\left(M_{h} \mid G\right)$ to score rule $P \Rightarrow y$ with respect to group $G$. We denote this Bayesian score by $\boldsymbol{B} \boldsymbol{S}(\boldsymbol{P} \Rightarrow \boldsymbol{y}, \boldsymbol{G})$.

Example 2. Let us use the Bayesian score to evaluate rule $R_{2}$ : Family history $=\mathrm{Yes} \Rightarrow \mathrm{CHD}$ in Example 1. We evaluate $R_{2}$ with respect to the entire dataset $G_{\phi}$ by computing $B S\left(R_{2}, G_{\phi}\right)$. Using the notations introduced earlier, $N_{* 1}=50$ and $N_{* 2}=100$ (the number of cases and controls in the dataset). Also, $N_{11}=30, N_{12}=20, N_{21}=N_{* 1}-N_{11}=20$ and $N_{22}=N_{* 2}-N_{12}=80$. Let us use uniform beta priors for all parameters: $\alpha=\beta=\alpha_{1}=\beta_{1}=\alpha_{2}=\beta_{2}=1$. The likelihood of $M_{e}$ is $3.2 \times 10^{-43}$, the likelihood of $M_{h}$ is $1.5 \times 10^{-38}$ and the likelihood of $M_{l}$ is $1 \times 10^{-44}$. Hence, $B S\left(R_{2}, G_{\phi}\right)=\operatorname{Pr}\left(M_{h} \mid G_{\phi}\right)=0.99998$. This implies that there is a strong evidence in the data to conclude that pattern Family history=yes makes CHD more likely.

\subsection{The Predictive and Non-Spurious Rules Score}

The Bayesian score proposed in the previous section provides a way to evaluate the predictiveness of a rule by contrasting it to a more general population than the population covered by the rule. One approach to supervised descriptive rule discovery is to score each rule $R_{i}$ with respect to the entire data $B S\left(R_{i}, G_{\phi}\right)$ and report the top rules to the user. However, this approach does not overcome the spurious rules problem: if a rule $P \Rightarrow y$ achieves a very high score, many spurious rules $P^{\prime} \Rightarrow y: P^{\prime} \supset P$ are expected to have a high score as well (provided that $P^{\prime}$ have enough support in the data). As a result, the rules presented to the user would contain a lot of redundancies and fail to provide a good coverage of the data.

To overcome this problem, we propose the Predictive and Non-Spurious Rules score, denoted as PNSR-score, which we define as follows:

$$
P N S R \text {-score }(P \Rightarrow y)=\min _{S: S \subset P}\left\{B S\left(P \Rightarrow y, G_{S}\right)\right\}
$$

If a rule $R$ achieves a high $P N S R$-score, then there is a strong evidence in the data not only to conclude that $R$ improves the prediction of its consequent with respect to the entire data, but also with respect to the data matching any of its subrules. That is, the rule's effect on the class distribution cannot be explained by any more general rule that covers a larger population. This implies that every item in the condition of the rule is an important contributor to its predictiveness (the rule is concise).

Example 3. Let us go back to Example 1 and compute the PNSR-score for rule $R_{3}$. If we evaluate $R_{3}$ with respect the entire dataset, $B S\left(R_{3}, G_{\phi}\right)=0.9997$. If we evaluate $R_{3}$ with respect to subrule $R_{1}, B S\left(R_{3}, G_{R_{1}}\right)=0.999992$. Finally, if we evaluate $R_{3}$ with respect to subrule $R_{2}, B S\left(R_{3}, G_{R_{2}}\right)=0.47$. We can see that $R_{3}$ is considered very predictive when compared to the entire dataset or to subrule $R_{1}$, but is not predictive when compared to subrule $R_{2}$. Therefore, we do not consider $R_{3}$ an important rule because it is equivocal whether it predicts CHD as being more likely than does $R_{2}$. 
Example 4. Let us consider again the simple Bayesian network in Figure 2 Assume we have 10 binary features $\left(F_{1}\right.$ to $\left.F_{10}\right)$ and the CPTs are defined as follows: $\operatorname{Pr}\left(F_{i}=1\right)=0.4: i \in\{1, \ldots, 10\}, \operatorname{Pr}\left(Y=y_{1} \mid F_{1}=1\right)=0.9$ and $\operatorname{Pr}\left(Y=y_{1} \mid F_{1}=0\right)=0.5$. Let the data $D$ be 500 instances that are randomly generated from this network and let us use $D$ to mine rules that are predictive of class $y_{1}$. As we discussed earlier, the only important rule for predicting $y_{1}$ is $F_{1}=1 \Rightarrow y_{1}$ and all other rules are just spurious.

We use frequent pattern mining to explore all patterns that occur in more than $10 \%$ of the data. Doing so, we obtain 1,257 frequent patterns (potential rules). If we apply the $\chi^{2}$ test with significance level $\alpha=0.05$, we get 284 rules that positively predicts $y_{1}$ and are statistically significant. Even if we apply the False Discovery Rate (FDR) technique [4] to correct for multiple hypothesis testing, we get 245 positive significant rules! If we use our Bayesian score to evaluate each rule (individually) with respect to the entire dataset and report rules with $B S\left(R_{i}, G_{\phi}\right) \geq 0.95$, we get 222 rules 5 . Note that this approach still suffers from the spurious rules problem. Let us now apply the confidence improvement constraint to filter out "non-productive" rules [3, 15, 19, 20, 26]. By doing so, we get 451 rules! This clearly demonstrates that the confidence improvement constraint is ineffective for removing spurious rules. Lastly, let us use our proposed $P N S R$-score and report rules with PNSR-score $\left(R_{i}\right) \geq 0.95$. Doing so, we obtain only a single rule $F_{1}=1 \Rightarrow y_{1}$ (the only important rule) and effectively filter out all other spurious rules.

\subsection{The Mining Algorithm}

In this section, we present the algorithm for mining predictive and non-spurious rules. The algorithm utilizes frequent pattern mining to explore the space of potential rules and applies the PNSR-score to evaluate the rules.

To search for rules, we partition the data according to the class labels $y \in$ $\operatorname{dom}(Y)$ and mine frequent patterns for each class separately (using a local minimum support $\sigma_{y}$ that is related to the number of instances from class $y$ ). The reason for doing this as opposed to mining frequent patterns from the entire data is that when the data is unbalanced, exploring only patterns that are globally frequent may result in missing many important rules for the rare classes.

The mining algorithm takes as input 1) the data instances from class $y: D_{y}=$ $\left.\left\{\left(x_{i}, y_{i}\right): y_{i}=y\right\}, 2\right)$ the data instances that do not belong to class $y: D_{\neg y}=$ $\left.\left\{\left(x_{i}, y_{i}\right): y_{i} \neq y\right\}, 3\right)$ the local minimum support threshold $\sigma_{y}$ and 4) a user specified significance parameter $g$. The algorithm explores the space of frequent patterns and outputs the rules with PNSR-score higher than $g$.

A straightforward way to obtain the result is to apply the commonly used two-phase approach as in [6, 10, 12, 17, 20, 26, 27], which generates all frequent patterns in the first phase and evaluates them in the second phase (a postprocessing phase). That is, we need to perform the following two steps:

${ }^{4}$ The prior of $y_{1}$ in this network is $\operatorname{Pr}\left(Y=y_{1}\right)=0.66$.

${ }^{5}$ The 0.95 threshold is chosen so that it is comparable to the commonly used frequentist 0.05 significance level. 
1. Phase I: Mine all frequent patterns: $F P=\left\{P_{1}, \ldots, P_{m}: \sup \left(P_{i}\right) \geq \sigma_{y}\right\}$

2. Phase II: For each pattern $P_{i} \in F P$, output rule $P_{i} \Rightarrow y$ if $P N S R$-score $\left(P_{i} \Rightarrow\right.$ $y) \geq g$.

In contrast to this two-phase approach, our algorithm integrates rule evaluation with frequent pattern mining, which allows us to apply additional pruning techniques that are not applicable in the two-phase approach.

The mining algorithm explores the lattice of frequent patterns level by level from the bottom-up starting from the empty pattern. That is, the algorithm first explores frequent 1-patterns, then frequent 2-patterns, and so on. When the algorithm visits a frequent pattern $P$ (a node in the lattice), it computes the $P N S R$-score of rule $P \Rightarrow y$ and adds it to result if $P N S R$-score $(P \Rightarrow y) \geq g$.

Lossless Pruning. We now illustrate how to utilize the PNSR-score to prune portions of the search space that are guaranteed not to contain any result.

We say that pattern $P$ is pruned if we do not explore any of its superpatterns $\left(P^{\prime} \supset P\right)$. This means that we exclude the entire sublattice with bottom $P$ from the lattice of patterns we have to explore.

Frequent pattern mining relies only on the support of the patterns to prune infrequent patterns according to the following anti-monotone property: if a pattern is not frequent, all of its superpatterns are guaranteed not to be frequent.

By integrating rule evaluation with frequent pattern mining, we can apply an additional pruning technique. The idea is to prune pattern $P$ if we guarantee that none of its superpatterns will be in the result:

$$
\text { Prune } P \text { if } \forall P^{\prime} \supset P: P N S R \text {-score }\left(P^{\prime} \Rightarrow y\right)<g
$$

However, since patterns are explored in a level-wise fashion, we do not know the class distribution in the superpatterns of $P$. But we know that for any $P^{\prime} \supset P$ : $G_{P^{\prime}} \subseteq G_{P}$, and hence $\sup \left(P^{\prime}, D_{y}\right) \leq \sup \left(P, D_{y}\right) \wedge \sup \left(P^{\prime}, D_{\neg y}\right) \leq \sup \left(P, D_{\neg y}\right)$.

We now define the optimal superpattern of $P$ with respect to class $y$, denoted as $P^{*}$, to be a hypothetical pattern that covers all instances from $y$ and none of the instances from the other classes:

$$
\sup \left(P^{*}, D_{y}\right)=\sup \left(P, D_{y}\right) \wedge \sup \left(P^{*}, D_{\neg y}\right)=0
$$

$P^{*}$ is the best possible superpattern for predicting $y$ that $P$ can generate. Therefore, $P N S R$-score $\left(P^{*} \Rightarrow y\right)$ is an upper bound on the PNSR-score for the superpattern of $P$. Now, we safely prune $P$ when $P N S R$-score $\left(P^{*} \Rightarrow y\right)<g$.

\section{Experimental Evaluation}

The experiments compare the performance of different rule quality measures for the problem of supervised descriptive rule discovery. In particular, we compare the following measures: 
1. GR: Rules are ranked using the Growth Rate measure, which was used in [1] in the context of emerging pattern mining.

$$
G R(P \Rightarrow y)=\frac{\sup \left(P, D_{y}\right) /\left|D_{y}\right|}{\sup \left(P, D_{\neg y}\right) /\left|D_{\neg y}\right|}
$$

where $D_{y}$ and $D_{\neg y}$ represent the instances from class $y$ and not from class $y$, respectively.

2. J-measure: Rules are ranked using the J-measure [25], a popular information theoretic measure that scores the rules by their information content.

$$
J \text {-measure }(P \Rightarrow y)=\frac{\sup (P, D)}{|D|} \times \sum_{z \in\{y, \neg y\}} \operatorname{conf}(P \Rightarrow z) \cdot \log _{2}\left(\frac{\operatorname{conf}(P \Rightarrow z)}{\operatorname{conf}(\Phi \Rightarrow z)}\right)
$$

3. WRAcc: Rules are ranked using the Weighted Relative Accuracy, which was used in [17] in the context of subgroup discovery 6 .

$$
W R A c c(P \Rightarrow y)=\frac{\sup (P, D)}{|D|} \times(\operatorname{conf}(P \Rightarrow y)-\operatorname{conf}(\Phi \Rightarrow y))
$$

Note that this measure is compatible (provides the same rule ranking) with the support difference heuristic used in [2] for contrast set mining (see [23]).

4. $B S$ : Rules are ranked using our proposed Bayesian score. However, this method scores each rule individually with respect to the entire data and do not filter out spurious rules.

5. Conf-imp: Only rules that satisfy the confidence improvement constraint are retained [3, 15, 19, 20, 26] and they are ranked according to their confidence.

6. PNSR: Only rules $R_{i}$ that have a PNSR-score $\left(R_{i}\right) \geq 0.95$ are retained 7 and they are ranked according to the Bayesian score.

Note that the $G R$ measure does not consider the coverage of the rule when assessing its interestingness. For example, $G R$ favors a rule that covers $8 \%$ of the instances of in one class and $1 \%$ of the instances in the other classes over a rule that covers $70 \%$ of the instances of in one class and $10 \%$ of the instances in the other classes (as $\frac{8}{1}>\frac{70}{10}$ ). As a result, $G R$ often chooses rules that are very specific (with low coverage) and do not generalize well. To overcome this, the J-measure and WRAcc explicitly incorporate the rule coverage $\frac{\operatorname{Sup}(P, D)}{|D|}$ in their evaluation functions to favor high coverage rules over low coverage rules. This is done by multiplying the rule coverage with a factor that quantifies the distributional surprise (unusualness) of the class variable in the rule (the cross entropy for $J$-measure and the relative accuracy for WRAcc). However, it is not clear whether simply multiplying these two factors leads to the optimal tradeoff. On the other hand, $B S$ achieves this trade-off automatically by modeling the

\footnotetext{
${ }^{6}$ The algorithm by [17] uses weighted sequential covering and modifies the WRAcc measure to handel example weights.

7 The 0.95 threshold is chosen so that it is comparable to the commonly used frequentist 0.05 significance level.
} 
uncertainty of the estimation (the more data we have, the more certain we are about the estimated probabilities).

Note that the first four methods ( $G R, J$-measure, WRAcc and $B S$ ) evaluate each rule individually with respect to the entire data and do not consider the nested structure of rules. On the other hand, conf-imp and PNSR evaluate each rule with respect to all of its subrules. Conf-imp simply requires each rule have a higher confidence than its subrules, while $P N S R$ requires each rule to show a substantial evidence that it improves the prediction over its subrules, which is evaluated using our proposed PNSR-score.

For all methods, we use frequent pattern mining to explore the space of potential rules and we set the local minimum support $\left(\sigma_{y}\right)$ to $10 \%$ the number of instance in the class. For $B S$ and PNSR, we use uniform beta priors (uninformative priors) for all parameters.

\subsection{Datasets}

We evaluate the performance of the different rule quality measures on 15 public datasets from the UCI Machine Learning repository. We discretize the numeric attributes into intervals using Fayyad and Irani discretization [13]. Table 1] shows the main characteristics of the datasets.

Table 1. UCI Datasets characteristics

\begin{tabular}{|c|c|c|c|}
\hline dataset & \# features & \# instances & \# classes \\
\hline \hline Lymphography & 18 & 142 & 2 \\
\hline Parkinson & 22 & 195 & 2 \\
\hline Heart & 13 & 270 & 2 \\
\hline Hepatitis & 19 & 155 & 2 \\
\hline Diabetes & 8 & 768 & 2 \\
\hline Breast cancer & 9 & 286 & 2 \\
\hline Nursery & 8 & 12,630 & 3 \\
\hline Red wine & 11 & 1,599 & 3 \\
\hline Mammographic & 5 & 961 & 2 \\
\hline Tic tac toe & 9 & 958 & 2 \\
\hline Ionosphere & 34 & 351 & 2 \\
\hline Kr vs kp & 36 & 3,196 & 2 \\
\hline Pen digits & 16 & 10,992 & 10 \\
\hline Zoo & 16 & 74 & 3 \\
\hline WDBC & 30 & 569 & 2 \\
\hline
\end{tabular}

\subsection{Quality of Top-K Rules}

For a set of rules to be practically useful, the rules should be accurate to predict the class label of unseen data instances (high precision) and the rule set should provide a good coverage of the data (high recall). 

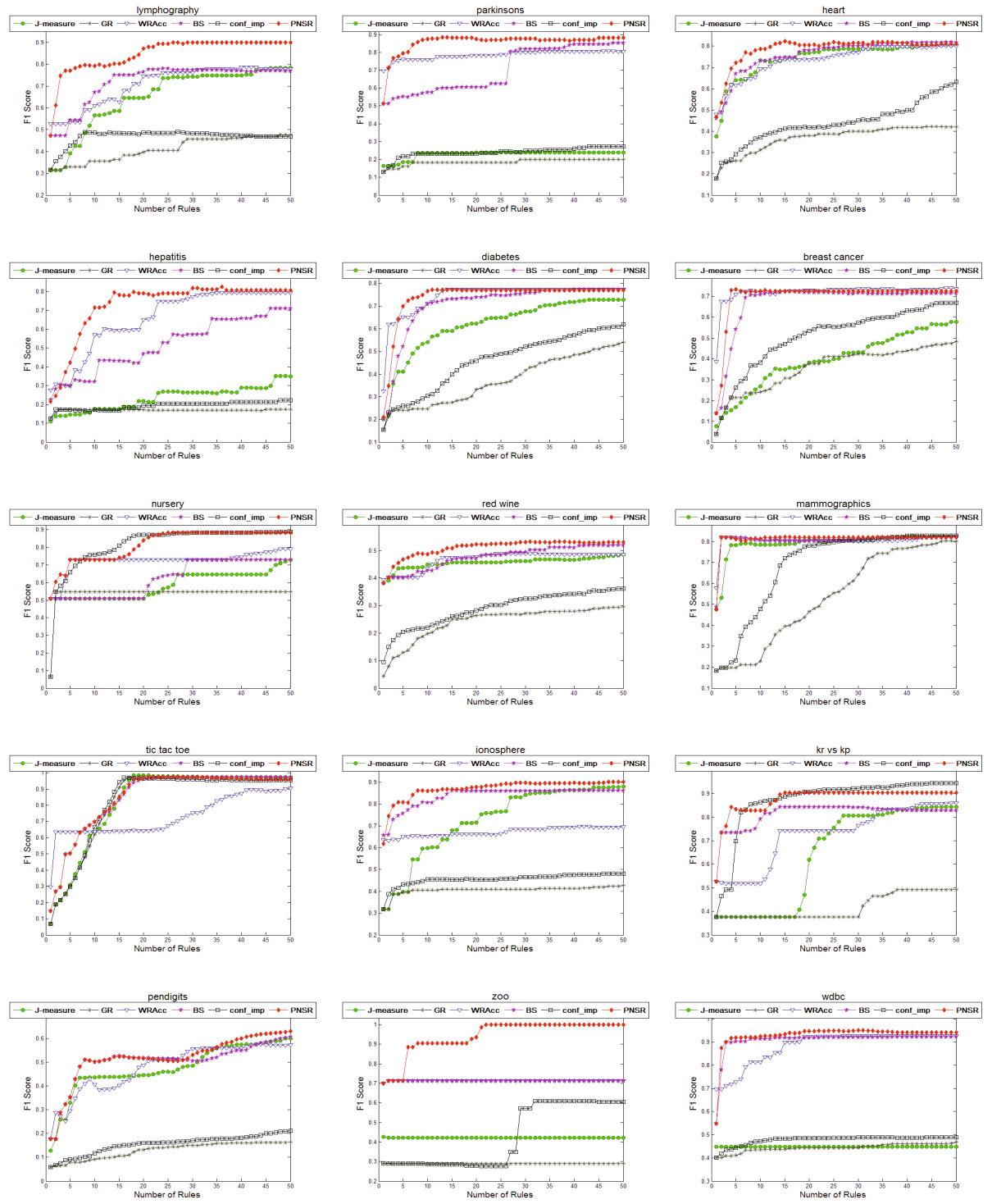

Fig. 4. Comparison of the performance of several rule evaluation measures. The X-axis is the number of the top rules and the Y-axis is the F1 score of the rule set. 
In this section, we compare the different rule evaluation measures according to the quality of the top rules. In particular, for each of the compared evaluation measures, we mine the top $k$ rules from the training data and use them to classify the testing data. The classification is done according to the highest confidence rule [21]:

$$
\operatorname{Prediction}(x)=\underset{y_{i}}{\arg \max }\left\{\operatorname{conf}\left(P \Rightarrow y_{i}\right): P \in x\right\}
$$

The classification performance is evaluated using the F1 score 24], which is the harmonic mean of the precision and recall. All results are reported using a 10-fold cross-validation scheme, where we use the same train/test splits for all compared methods.

Figure 4 shows the classification performance for the different number of top rules. We can see that $G R$ is the worst performing method for most datasets. The reason is that rules with the highest $G R$ scores are usually very specific (low coverage) and may easily overfit the training data. The other measures $(J$-measure, WRAcc and $B S$ ) perform better than $G R$ because they favor highcoverage rules over low-coverage rules, which results in rules that generalize better on the testing data. However, because these measures do not consider the relations among the rules, the top rules contain many spurious rules (rules describing the same underlying pattern and are small variations of each other). As a result, they fail to provide a good coverage of the data (see for example the lymphography and the zoo datasets). Finally, we can see that for most datasets, PNSR achieves the best performances with the smallest number of rules.

\subsection{Mining Efficiency}

In this section, we study the efficiency of our mining algorithm. In particular, we compare the running time of the following methods:

1. FPM: Frequent patterns mining, where we partition the data according to the class label and mine frequent patterns for each class (see Section 3.4). We apply the algorithm by [29], which mines frequent patterns using the vertical data format.

2. PNSR-Naive: The naive two-phase implementation for mining predictive and non-spurious rules, which applies FPM to mine all frequent patterns and then computes the PNSR-score of the patterns.

3. PNSR: Our mining algorithm, which integrates rule evaluation with frequent pattern mining and applies the lossless pruning technique described in Section 3.4 to prune the search space.

The running time is measured on a Dell Precision T1600 machine with an Intel Xeon $3 \mathrm{GHz}$ CPU and $16 \mathrm{~GB}$ of RAM. As before, we set the local minimum support $\left(\sigma_{y}\right)$ to $10 \%$ the number of instance in the class. Table 2 shows the execution time (in seconds) of the compared methods on the UCI datasets.

The results show that on seven of the fifteen datasets (lymphography, Parkinson, Heart, Hepatitis, Ionosphere, Zoo and WDBC), PNSR is more efficient than $F P M$, which is the cost of the first phase of any two-phase method 
Table 2. The execution time (in seconds) of frequent pattern mining (FPM), twophase PNSR mining (PNSR-Naive) and our mining algorithm (PNSR)

\begin{tabular}{|c||c|c|c|}
\hline dataset & $F P M$ & PNSR-Naive & PNSR \\
\hline \hline Lymphography & 328 & 410 & 153 \\
\hline Parkinson & 9,865 & 11,229 & 800 \\
\hline Heart & 45 & 69 & 37 \\
\hline Hepatitis & 1,113 & 1,284 & 391 \\
\hline Diabetes & 3 & 5 & 5 \\
\hline Breast cancer & 3 & 5 & 4 \\
\hline Nursery & 2 & 9 & 9 \\
\hline Red wine & 28 & 52 & 50 \\
\hline Mammographic & 1 & 1 & 1 \\
\hline Tic tac toe & 3 & 4 & 4 \\
\hline Ionosphere & 16,899 & 19,765 & 1,077 \\
\hline Kr vs kp & 1,784 & 2,566 & 2,383 \\
\hline Pen digits & 71 & 144 & 138 \\
\hline Zoo & 185 & 244 & 23 \\
\hline WDBC & 2,348 & 4,320 & 282 \\
\hline
\end{tabular}

6. 10, 12, 17, 20, 26, 27]. For some of these datasets, PNSR drastically improves the efficiency. For example, on the Parkinson, Ionosphere datasets, PNSR is more than an order of magnitude faster than FPM. This shows that utilizing the predictiveness of patterns to prune the search space can greatly help improving the mining efficiency.

\section{Conclusion}

In this paper, we study the problem of supervised descriptive rule discovery and propose a new rule evaluation score, the Predictive and Non-Spurious Rules (PNSR) score. This score relies on Bayesian inference to measure the quality of the rules. It also considers the structure of the patterns to ensure that each rule in the result offers a significant predictive advantage over all of its generalizations. We present an algorithm for mining rules with high PNSR scores, which efficiently integrates rule evaluation with frequent pattern mining. The experimental evaluation shows that our method is able to explain and cover the data with fewer rules than existing methods, which is beneficial for knowledge discovery.

Acknowledgments. This work was supported by grants 1R01GM088224-01 and 1R01LM010019-01A1 from the NIH. Its content is solely the responsibility of the authors and does not necessarily represent the official views of the NIH.

\section{References}

1. Agrawal, R., Srikant, R.: Fast algorithms for mining association rules in large databases. In: Proceedings of the International Conference on Very Large Data Bases, VLDB (1994) 
2. Bay, S.D., Pazzani, M.J.: Detecting group differences: Mining contrast sets. Data Mining and Knowledge Discovery 5, 213-246 (2001)

3. Bayardo, R.J.: Constraint-based rule mining in large, dense databases. In: Proceedings of the International Conference on Data Engineering, ICDE (1999)

4. Benjamini, Y., Hochberg, Y.: Controlling the false discovery rate: A practical and powerful approach to multiple testing. Journal of the Royal Statistical Society 57(1), 289-300 (1995)

5. Brin, S., Motwani, R., Silverstein, C.: Beyond market baskets: generalizing association rules to correlations. In: Proceedings of the International Conference on Management of Data, SIGMOD (1997)

6. Cheng, H., Yan, X., Han, J., Wei Hsu, C.: Discriminative frequent pattern analysis for effective classification. In: Proceedings of the International Conference on Data Engineering, ICDE (2007)

7. Clark, P., Niblett, T.: The cn2 induction algorithm. Machine Learning (1989)

8. Cohen, W.: Fast effective rule induction. In: Proceedings of International Conference on Machine Learning, ICML (1995)

9. Cohen, W., Singer, Y.: A simple, fast, and effective rule learner. In: Proceedings of the National Conference on Artificial Intelligence, AAAI (1999)

10. Deshpande, M., Kuramochi, M., Wale, N., Karypis, G.: Frequent substructurebased approaches for classifying chemical compounds. IEEE Transactions on Knowledge and Data Engineering 17, 1036-1050 (2005)

11. Dong, G., Li, J.: Efficient mining of emerging patterns: discovering trends and differences. In: Proceedings of the International Conference on Knowledge Discovery and Data Mining, SIGKDD (1999)

12. Exarchos, T.P., Tsipouras, M.G., Papaloukas, C., Fotiadis, D.I.: A two-stage methodology for sequence classification based on sequential pattern mining and optimization. Data and Knowledge Engineering 66, 467-487 (2008)

13. Fayyad, U., Irani, K.: Multi-interval discretization of continuous-valued attributes for classification learning. In: Proceedings of the International Joint Conference on Artificial Intelligence, IJCAI (1993)

14. Geng, L., Hamilton, H.J.: Interestingness measures for data mining: A survey. ACM Computing Surveys 38 (2006)

15. Grosskreutz, H., Boley, M., Krause-Traudes, M.: Subgroup discovery for election analysis: a case study in descriptive data mining. In: Proceedings of the International Conference on Discovery Science (2010)

16. Heckerman, D., Geiger, D., Chickering, D.M.: Learning bayesian networks: The combination of knowledge and statistical data. Machine Learning (1995)

17. Kavsek, B., Lavrač, N.: APRIORI-SD: Adapting association rule learning to subgroup discovery. Applied Artificial Intelligence 20(7), 543-583 (2006)

18. Lavrač, N., Gamberger, D.: Relevancy in Constraint-Based Subgroup Discovery. In: Boulicaut, J.-F., De Raedt, L., Mannila, H. (eds.) Constraint-Based Mining. LNCS (LNAI), vol. 3848, pp. 243-266. Springer, Heidelberg (2006)

19. Li, J., Shen, H., Topor, R.: Mining Optimal Class Association Rule Set. In: Cheung, D., Williams, G.J., Li, Q. (eds.) PAKDD 2001. LNCS (LNAI), vol. 2035, p. 364. Springer, Heidelberg (2001)

20. Li, W., Han, J., Pei, J.: CMAR: Accurate and efficient classification based on multiple class-association rules. In: Proceedings of the International Conference on Data Mining, ICDM (2001)

21. Liu, B., Hsu, W., Ma, Y.: Integrating classification and association rule mining. In: Knowledge Discovery and Data Mining, pp. 80-86 (1998) 
22. Nijssen, S., Guns, T., De Raedt, L.: Correlated itemset mining in roc space: a constraint programming approach. In: Proceedings of the International Conference on Knowledge Discovery and Data Mining, SIGKDD (2009)

23. Novak, P.K., Lavrač, N., Webb, G.I.: Supervised descriptive rule discovery: A unifying survey of contrast set, emerging pattern and subgroup mining. Journal of Machine Learning Research (JMLR) 10, 377-403 (2009)

24. Sebastiani, F.: Machine learning in automated text categorization. ACM Computing Surveys (2002)

25. Smyth, P., Goodman, R.M.: An information theoretic approach to rule induction from databases. IEEE Transactions on Knowledge and Data Engineering (1992)

26. Webb, G.I.: Discovering significant patterns. Machine Learning 68(1), 1-33 (2007)

27. Xin, D., Cheng, H., Yan, X., Han, J.: Extracting redundancy-aware top-k patterns. In: Proceedings of the International Conference on Knowledge Discovery and Data Mining, SIGKDD (2006)

28. Yang, Y., Webb, G.I., Wu, X.: Discretization methods. In: The Data Mining and Knowledge Discovery Handbook, pp. 113-130. Springer (2005)

29. Zaki, M.J.: Scalable algorithms for association mining. IEEE Transaction on Knowledge and Data Engineering (TKDE) 12, 372-390 (2000) 